\title{
Evading immune escape: synergy of COX and immune-checkpoint inhibitors
}

Tumour development is associated with an immunoediting process, whereby the immune system selects the outgrowth of less immunogenic tumours. Santiago Zelenay and coauthors were interested in eludicating the mechanisms that enable cancer cells to evade antitumour immunity. "Our interest was triggered by the compelling evidence that the immune system can act as a barrier to cancer, as noted by the remarkable success obtained over the past few years with certain types of immunotherapies."

The researchers cultured genetically engineered cancer cell lines with innate immune cells to identify modulatory factors that subvert innate immune cell function. CRISPR-Cas9 technology was used to ablate the ability of several types of cancer cell lines to produce prostaglandin E2 $\left(\mathrm{PGE}_{2}\right)$, which possesses potent immunomodulatory properties in vitro and in vivo that enable immune escape of tumours and their progressive growth. The researchers inhibited $\mathrm{PGE}_{2}$ production in vivo by administering cyclooxygenase (COX) inhibitors to assess if this treatment could increase the efficacy of anti-PD-1 blockade. They then analysed publicly available human melanoma datasets to look for evidence of $\mathrm{PGE}_{2}$-dependent immune modulation in humans.

"The most significant finding is that cancer cell-derived $\mathrm{PGE}_{2}$ fuels cancerpromoting inflammation and allows escape from type I interferon and T-celldependent tumour elimination. This escape can be reversed by the combination of immunotherapy with COX inhibitors." The results highlight that cancer cells do not pass unnoticed by the immune system, but actively evade antitumour immunity; crucially, $\mathrm{PGE}_{2}$ production enables a major mechanism of immune escape.

Zelenay concludes: "our work has clear therapeutic implications. These preliminary data suggest that COX inhibitors could act synergistically with immune-checkpoint blockade and potentially other types of

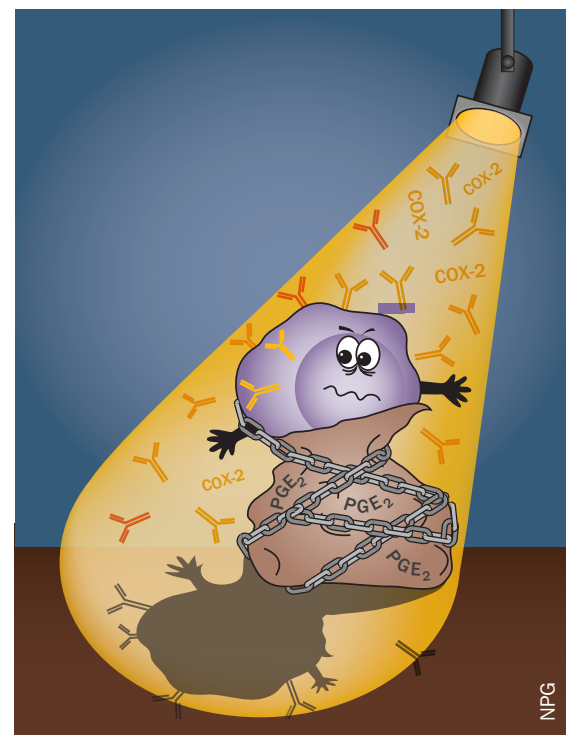

cancer therapies. Our data also indicate that COX-2 expression might constitute a useful biomarker of unresponsiveness to immunotherapy, and that the expression of specific inflammatory signatures in patient biopsy samples might help predict treatment outcome." These results lay the foundation for setting up clinical trials to test the efficacy and safety of the combination of COX inhibitors with other therapies for cancer treatment.

\section{Lisa Hutchinson}

Original article Zelenay, S. et al. Cyclooxygenasedependent tumor growth through evasion of immunity. Cell doi:10.1016/j.cell.2015.08.015 